\title{
Optimized antithymocyte globulin dose in high risk kidney transplantation
}

\author{
Sangkyun Mok, Young Jun Park, Wonjong Kim, Boyoon Choi, Sun Cheol Park, Sang Seob Yun
}

Department of Surgery-Transplantation, The Catholic University of Korea, Seoul St. Mary's Hospital, Seoul, Korea

Background: Antithymocyte globulin (ATG) induction therapy is important for graft survival in high-risk recipients of kidney transplantation. However, the optimal dose of ATG is controversial. The purpose of our study was to analyze the optimal dose of ATG in high-risk kidney transplantation.

Methods: This retrospective study included 801 consecutive patients from a prospectively registered database who underwent kidney transplantation at Seoul St. Mary's Hospital, South Korea, between January 1, 2019, and December 30, 2020. Receiver operating characteristic (ROC) curve analysis was used to confirm the optimized cut-off dose associated with graft survival. Comparison analysis between the two ATG dose groups was performed. Death-censored graft loss and mortality were analyzed using the Kaplan-Meier method.

Results: A total of 771 patients were enrolled in the study (mean age, $48.7 \pm 11.5$ years; range, $16-75$ years). Of these, 445 patients (57.7\%) was male and 326 (42.3\%) was female; 157 patients (20.4\%) underwent ABO incompatible kidney transplantation and 196 (25.4\%) underwent deceased donor kidney transplantation. Using ROC curve analysis, the optimized total ATG dose associated with graft survival was found to be $287.76 \mathrm{mg}$ (AUC, 0.644; $\mathrm{P}=0.027$; Youden index, 0.309).

Conclusions: In our study, it was suggested that the use of more than $4.78 \mathrm{mg} / \mathrm{kg}$ total dose ATG was associated with good graft survival in high-risk kidney transplantation recipients. Therefore, appropriate ATG administration is required for these patients.

Corresponding author: Sangkyun Mok

E-mail: skmok81@gmail.com

(c) The Korean Society for Transplantation

This is an Open Access article distributed under the terms of the Creative Commons Attribution Non-Commercial License (http://creativecommons.org/licenses/by-nc/4.0/) which permits unrestricted non-commercial use, distribution, and reproduction in any medium, provided the original work is properly cited. 